\title{
Clinical Evaluation of Dental Implants Performed in the Universitas Indonesia Dental Training Hospital Periodontal Clinic from 2009 to 2014
}

\author{
Faradina Putriyanti ${ }^{1}$, Yuniarti Soeroso ${ }^{2}$, Hari Sunarto ${ }^{2}$ \\ ${ }^{1}$ Periodontics Residence Program, Faculty of Dentistry, Universitas Indonesia, Jakarta, Indonesia \\ ${ }^{2}$ Department of Periodontia, Faculty of Dentistry, Universitas Indonesia, Jakarta, Indonesia \\ *Email: yuniarti_22@yahoo.co.id
}

\begin{abstract}
There is no long-term evaluation of the clinical conditions of dental implants performed in the Universitas Indonesia (UI) Dental Training Hospital periodontal clinic. The aim of this study is to evaluate the clinical condition of dental implants performed in the UI Dental Training Hospital periodontal clinic from 2009 to 2014. Eleven patients received 29 dental implants. The implants were placed from 2009 to 2014. Probing pocket depth, loss of attachment, bleeding on probing, gingival recession, implant mobility, and oral hygiene index were assessed in 2016. In our study, no implant mobility was found. The mean pocket depth was $2.43 \pm 1.7 \mathrm{~mm}$. The mean gingival recession was $0.24 \pm 0.47 \mathrm{~mm}$. The mean clinical attachment loss was $0.26 \pm 0.54 \mathrm{~mm}$. When dental implants were examined, $46.6 \%$ of them showed bleeding on probing, while $53.4 \%$ showed no bleeding on probing. There was no statistically significant relationship between different oral hygiene indexes and probing pocket depth, loss of attachment, or bleeding on probing. There was a statistically significant difference in gingival recession between patients with different oral hygiene indexes. Based on this study, it is concluded that dental implants performed in the UI Dental Training Hospital periodontal clinic can still provide satisfactory results after 2 to 7 years.
\end{abstract}

Keywords: dental implant, probing depth, bleeding on probing, mobility, recession

\section{Introduction}

Dental implant treatment is applied in dentistry practice for the treatment of partially or fully edentulous teeth [1]. The success rate of dental implants is higher than that of other tooth-supported dentures [2]. The frequent use of dental implants is due to the elderly living longer, failure of fixed dental prostheses, anatomical consequences of the edentulous area, the positive predicted long-term results of dental implants, and society's increased awareness about the advantages of dental implants [3].

The important criteria for the long-term success of dental implants are stable bone support, intermediate inflammation degree around the implant area, good aesthetics, and crown-supported implants with good function [4]. Other criteria for successful dental implants are no implant mobility, no subjective complaints from 
the patient, no peri-implantitis infection with recurrent suppuration, no radiolucency around the implant, a probing depth not more than $5 \mathrm{~mm}$, and no bleeding on probing [5]. The average time of dental implant evaluation is approximately 3 years post-implantation [6].

Dental implant treatment has been part of the specialist treatments offered by the UI Dental Training Hospital periodontal clinic since 2009. Currently, there are no long-term evaluation reports regarding the dental implants performed in the UI Dental Training Hospital periodontal clinic. The aim of this study was to evaluate the clinical condition of dental implants performed in the UI Dental Training Hospital periodontal clinic from 2009 to 2014 .

\section{Method}

The samples used in this study were 29 dental implants from patients of the UI Dental Training Hospital periodontal clinic. The implants were placed from 2009 to 2014. In 2016, probing pocket depth, loss of attachment, bleeding on probing, gingival recession, implant mobility, and oral hygiene index were assessed for each implant.

For probing pocket depth, a Colorvue Probe UNC 12 with a satin steel handle (Hu-Friedy, America) was used. The pocket depth was measured from the mesiobuccal site, buccal site, distobuccal site, mesiolingual site, lingual site, and distolingual site. Loss of attachment was measured from the crown margin to the base of the pocket Gingival recession was measured from the crown margin to the gingival margin. Implant mobility was checked manually and with a dental instrument. Bleeding on probing was measured by inserting a periodontal probe into the sulcus with light pressure in the buccal and lingual sites [7].

According to Greene and Vermilion (1960), the oral hygiene index is composed of the debris index and calculus index combined. Each index is based on 12 numerical determinations representing the amount of debris and calculus found on the buccal and lingual surfaces of all three segments of each dental arch. In this study, the clinical examination results are presented as means and standard errors. Associations between the oral hygiene index and clinical examinations were analyzed with Mann-Whitney $U$ tests and chi-squared tests.

\section{Results}

Data from 11 patients, 5 males and 6 females, were included in the evaluation. The age of the patients ranged from 24 years to 59 years (mean age, 44.3 years). The patients received 29 dental implants, 13 in the maxilla and 16 in the mandible. All 29 implants were successful, resulting in a cumulative survival rate of $100 \%$. All patients in this study maintained moderate to good oral hygiene. Of the 29 patients, $81.8 \%$ had good oral hygiene, and $18.20 \%$ patients had moderate oral hygiene. Clinical parameters such as probing depth, gingival recession, and clinical attachment loss are described in Table. 1. 
Table. 1 Mean values of clinical parameters at 2- to 7-year follow-up

\begin{tabular}{|c|c|c|}
\hline Clinical Parameters & Mean Loss (SD) (mm) & Range \\
\hline Probing depth & $2.43(1.70)$ & $0-7$ \\
\hline Gingival recession & $0.24(0.47)$ & $0-2$ \\
\hline Clinical attachment loss & $0.26(0.54)$ & $0-3$ \\
\hline
\end{tabular}

There was no statistically significant difference in probing depth between patients with different oral hygiene indexes (Mann-Whitney $U$ test: $p=0.3$ ). There was a statistically significant difference between different in gingival recession between patients with different oral hygiene indexes (Mann-Whitney U test: $p=0.01)$. There was no statistically significant difference in clinical attachment loss between patients with different oral hygiene indexes (MannWhitney U test: $p=0.05$ ). The percentage of patients who exhibited bleeding on probing is shown in Graph. 1.

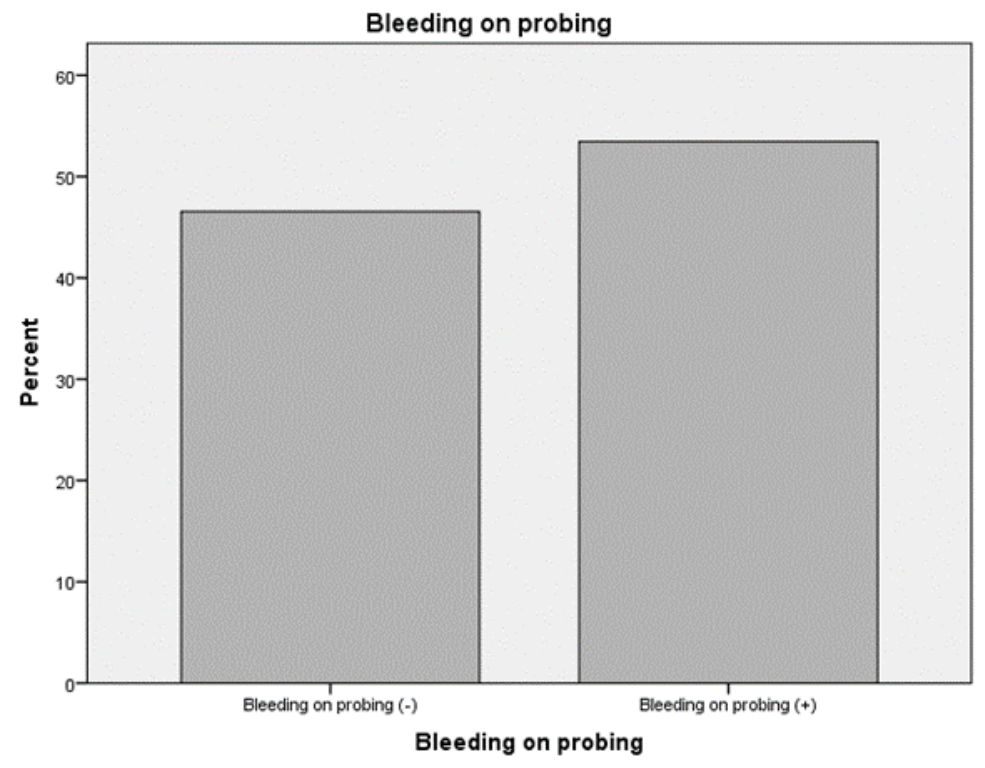

Graph. 1. Chart showing percentage of bleeding on probing

There was no statistically significant difference in bleeding on probing the gingiva between patients with different oral hygiene indexes (chi-squared test: $p=$ 0.74).

\section{Discussion}

The aim of this study was to evaluate the dental implant treatment outcomes of patients of the UI Dental Training Hospital periodontal clinic 2-7 years after implantation. For the survival and success of implants to be analyzed appropriately, a minimum of 5 years of consistent follow-up is necessary [8]. In 
this study, 29 dental implants were first evaluated 2-7 years (placed from 2009 to 2014) after implantation because no long-term records were available. The implants were recalled in 2016 as part of a routine annual recall program. The implants were functional and clinically stable when individually tested. There was no implant mobility, and there were no subjective complaints. Implant mobility is an indication of lack of osseointegration. It is of no use in diagnosing early implant disease but shows the final stages of de-integration [9].

The success rate of the inserted implants was $100 \%$, which is comparable with those of other long-term follow-up studies [10]. A 10-year study reported dental implant survival rates of $90 \%$ to $96.6 \%$ for 101 periodontally compromised and periodontally healthy patients [11]. However, the success of implant therapy is not only determined by high survival rates but also by stable hard- and soft-tissue conditions [12].

A two-year longitudinal study found that the probing attachment level and radiographic parameters together serve as a good predictor of peri-implant tissue status [13]. Successful implants generally allow a probe penetration of approximately $3 \mathrm{~mm} .6 \mathrm{In}$ this study, the average probing depth was $2.43 \pm 1.7$ $\mathrm{mm}$. This probing depth is considered normal for dental implants. 5 The mean value of periodontal probing depth in patients with good oral hygiene was less than that in patients with moderate oral hygiene, but the difference was not significant.

In another study, 6 months following insertion of a final prosthetic reconstruction, $38 \%-57 \%$ of implants demonstrated a peri-implant mucosal recession of $\geq 1 \mathrm{~mm}$. These recession measurements remained constant over a longer observation period of 16 months [14]. There was gingival recession in our study, but the mean measurement was only $0.24 \pm 0.47 \mathrm{~mm}$. There was a statistically significant difference between different oral hygiene indexes and gingival recession. Oates et al. (2002) evaluated long-term changes in soft-tissue height on the facial surface of 106 International Team for Implantology implants in 39 patients. They reported no implant failures after 2 years and $1 \mathrm{~mm}$ or more of soft-tissue recession around $61 \%$ of the implants [15].

The mean clinical attachment loss for this study was $0.26 \pm 0.54 \mathrm{~mm}$. There was no statistically significant difference between different oral hygiene indexes and clinical attachment loss (Mann-Whitney $U$ test: $p=0.05$ ). In all analyses, implants presented statistically significantly higher values of clinical attachment loss than teeth [16].

Biological complications are the main complications encountered when dealing with dental implants and are characterized by undesirable reactions in the implantsupporting tissues. The most common biological complication is mucositis, which is characterized by a reversible inflammatory process, demonstrated color change, and redness and bleeding of the mucosa around the implants, with no signs of bone resorption.8 Bleeding on probing of the gingiva was shown in $53.4 \%$ of dental implants. Patients with good oral hygiene showed less bleeding on probing than those with moderate oral hygiene. There was no statistically significant difference in bleeding on probing of the gingiva between different oral hygiene indexes. It is important to consider that plaque accumulation on implants or 
abutment surfaces induces a gingival inflammatory reaction [17]. Another study found no correlation between bleeding on probing and histologic, microbiologic, or radiographic changes around implants. They hypothesized that bleeding could have been caused by inappropriate force transmission from the periodontal probe tip to the peri-implant soft tissues [7]. The implants in another study presented double the frequency of bleeding, an undesired sign of inflammation, compared with teeth. The predictive meaning of bleeding on probing is still not well known [16]. Therefore, periodic recording of bleeding on probing in conjunction with measurement of probing depth is recommended for monitoring the condition of peri-implant soft tissues [18].

\section{Conclusion}

Good oral hygiene, in addition to proper patient selection, treatment planning, and implant placement, is very important in reducing the risks of peri-implant infections and increased probing pocket depth. Dental implantations performed in the UI Dental Training Hospital periodontal clinic have satisfactory results after 2 to 7 years. The lack of standardized and internationally recognized success criteria makes it difficult to compare the results of this study to those of other studies. In this study, we used the modified criteria created by Albrektsson et al. (1986). Systematic and continuous monitoring of peri-implant tissues during maintenance care is recommended for the early diagnosis of peri-implant disease.

\section{References}

1. Park W, Park Y, Park H, Yoo S, Chung S, Han J, Kim SW, Kim DM. A 10-year retrospective radiographic study of implantium dental implants. Int J Periodontics Restorative Dent. 2015;35(1).

2. Hämmerle $\mathrm{CH}$, Glauser R. Clinical evaluation of dental implant treatment. Periodontology 2000. 2004;34(1):230-9.

3. Misch C, Hamsah A. Contemporary Implant Dentistry. Contemp. Implant. Dent. Mosby Elsevier. 2008

4. Smith DE, Zarb GA. Criteria for success of osseointegrated endosseous implants. J Prosthet Dent. 1989;62(5):567-72.

5. Albrektsson T, Zarb G, Worthington P, Eriksson AR. The long-term efficacy of currently used dental implants: a review and proposed criteria of successInt J Oral Maxillofac Implants. 1986; 1(1):11-25.

6. Nemli SK, Güngör MB, Aydın C, Yılmaz H, Bal BT, Arıcı YK. Clinical and radiographic evaluation of new dental implant system: Results of a 3-year prospective study. J Dent Sci. 2016;11(1):29-34.

7. Salvi GE, Lang NP. Diagnostic parameters for monitoring peri-implant conditionsInt J Oral Maxillofac Implants. 2004;19(7).

8. Moraschini V, Poubel LD, Ferreira VF, dos Sp Barboza E. Evaluation of survival and success rates of dental implants reported in longitudinal studies with a follow-up period of at least 10 years: a systematic review. Int J Oral Maxillofac Surg. 2015;44(3):377-88

9. Chen S, Darby I. Dental implants: Maintenance, care and treatment of peri - implant infection. Aust Dent J. 2003;48(4):212-20. 
10. Moeintaghavi A, Radvar M, Arab HR, Boostani HR, Ghiami E. Evaluation of 3-to 8year treatment outcomes and success rates with 6 implant brands in partially edentulous patients. J Oral Implantol. 2012;38(S1):441-8.

11. Roccuzzo M, De Angelis N, Bonino L, Aglietta M. Ten - year results of a three - arm prospective cohort study on implants in periodontally compromised patients. Part 1: implant loss and radiographic bone loss. Clin Oral Implants Res. 2010;21(5):490-6.

12. Meijer HJ, Stellingsma K, Meijndert L, Raghoebar GM. A new index for rating aesthetics of implant - supported single crowns and adjacent soft tissues - the Implant Crown Aesthetic Index. Clin Oral Implants. 2005;16(6):645-9.

13. Brägger U, Hugel - pisoni C, Bürgin W, Buser D, Lang NP. Correlations between radiographic, clinical and mobility parameters after loading of oral implants with fixed partial dentures. A 2 - year longitudinal study. Clin Oral Implants Res. 1996;7(3):230-9.

14. Schwarz F, Mihatovic I, Shirakata Y, Becker J, Bosshardt D, Sculean A. Treatment of soft tissue recessions at titanium implants using a resorbable collagen matrix: a pilot study. Clin Oral Implants Res. 2014;25(1):110-5.

15. Oates TW, West J, Jones J, Kaiser D, Cochran DL. Long-term changes in soft tissue height on the facial surface of dental implants. Implant Dent. 2002;11(3):272-9.

16. Abreu MH, Bianchini MA, Magini RS, Rösing CK. Clinical and radiographic evaluation of periodontal and peri-implant conditions in patients with implant-supported prosthesis. Acta Odontol Latinoam. 2007;20(2):87-95.

17. Rasperini G, Maglione M, Cocconcelli P, Simion M. In vivo early plaque formation on pure titanium and ceramic abutments: a comparative microbiological and SEM analysisClin Oral Implants Res. 1998;9(6):357-64.

18. Todescan S, Lavigne S, Kelekis-Cholakis A. Guidance for the maintenance care of dental implants: clinical review. J Can Dent Assoc. 2012;78(1):107. 